\title{
Tracking of the LAZIO region shoreline from orthophotos AGEA 2014 and implementation of the database layer
}

\author{
Erik Biscotti $^{\mathrm{a}}$, Nicola Pizzeghello ${ }^{\mathrm{b}}$, Chiara Murri $^{\mathrm{c}}$, Graziano Colistra $^{\mathrm{d}}$ and Ilenia Batzu $^{\mathrm{e}}$ \\ ${ }^{a}$ Istituto Idrografico della Marina, Head of Standard \& Coordination Division, email: erikd.biscotti@marina.difesa.it \\ ${ }^{b}$ Istituto Idrografico della Marina, Head of Bathymetric \& Survey Division, email: nicolam.pizzeghello@marina.difesa.it \\ ${ }^{c}$ Istituto Idrografico della Marina, Head of Survey Section, email: chiara.murri@marina.difesa.it \\ ${ }^{d}$ Istituto Idrografico della Marina, Geo-topographic Section, email : graziano_colistra@marina.difesa.it \\ ${ }^{e}$ IHO Hydrographic Surveyor CAT A, Genova, email: ilenia.batzu@gmail.com
}

\begin{abstract}
The integrated coastal zone management (ICZM) is the modern approach used in the study, management and exploitation of the coastal area in various applications whereas in this area are concentrated interests concerning the most different fields, economic, environmental, legal, scientific and social. The coast is in fact inherently unstable by nature and consequently its characterization should take into account a continuous monitoring and up- dating of its variations and trends. The coastal area is that portion of land emerged and submerged containing the shoreline and is subject to both continental and marine geomorphic processes. The shoreline is the clearest expression of how this sector is particularly dynamic. Proper analysis and representation of the shape and nature of the coastal area are a first step to provide reliable and comparable tools to those who study and manage it. This paper presents the results of a study aimed to the realization of an integrated approach in the extraction of the shoreline using a case study of Lazio coast as a part of the European Project "Intercoast".
\end{abstract}

This work is based on national and international directives on the coastal zone, whether linked to a more terrestrial or maritime area, still within the broad definition of Hydrography provided by the International Hydrographic Organization (IHO).

The spatial information extracted by direct or indirect measurements of the most dynamic coastal sector emerged and submerged (emerged coast and sea bottom) have been provided by associating with a budget of measurement uncertainties, and assessing the quality.

\section{Introduction}

The Coastal Zone is an extremely complex area due to its anthropogenic and natural component and, at the same time, it is vulnerable and exploited for environmental, economic, social and defence interests.

Historically this interests are related to the Hydrographic world, which deals with the study and representation of territory and its evolution especially in the field of maritime navigation.

The Italian Navy Hydrographic Institute (hereinafter IIM), as national representing in international hydrographic Committee and Organizations such IHO (International Hydrographic Organization) in Italy, is heavily involved Authority in the survey of the Coastal Zone, where the safety of the navigation appears critical.

In the last decades the focus on the coastal band has been increased not only by the local regions, who are in Italy responsible to manage and protect this areas, but also by the central Environmental Ministry that is involved in European Environmental Policy. For this reason a number of Directives has been promulgated about environmental protection and study of territory, such INSPIRE, Marine Strategy etc., and most of them are oriented to the integrated coastal zone management.

\subsection{Aim of the study}

The study presented in this paper aim to explain an application of the above directives, concerning the collection and analysis of geographical data of the coastal zone as a part of a robust MSDI (Marine Spatial Data Infrastructure). Furthermore, this paper describes the result of the collaboration between the IIM and the Lazio Region with the Public Partnership Agreement - Project Regional Operative Program (ROP) of the European Regional Development Fund (ERDF) 2007-2013 "INTERCOAST" between the Lazio Region and the Italian Navy (2 November 2015), which had the following objective:

"To provide advanced procedures for the production of an official digital chart for the description of the coast- line and its topology, through the definition of appropriate standards for the validation and sharing of the existing data and for the development of a set of procedures for the acquisition, validation and testing of the new data ".

The jointed activities have been focused on:

"Shoreline Tracking and its typological-morphological characterization using AGEA ${ }^{1} 2014$ orthophotos".

\footnotetext{
1 AGenzia per le Erogazioni in Agricoltura: Agency for Agricultural Payments
} 
The geographical position of the shoreline and the extension of the coastal zone on the time scale are also important for the following reason:

"Land management in relation to the objective requirements (urbanization, tourism, hydro-geological, erosion, CC effects, etc.) and other activities results from specific European regulations (Floods Directive 60/2007 / EC, Planning Area Maritime Directive 89 / 2014 / EU, etc.)."

This work also aim to identify a shared procedure on shoreline tracking and its extrapolation from the analysis of aerial photographs. The result of this procedure will be completed by the implementation of the spatial database that is compliant with the above directives.

\section{Coastal measurement and Reference system}

All the measurements referred to the coastal zone are related to the shape and nature of the emerged and submerged coast, representing it through a spatial continuum without break lines with only methodological and measurement approach.

The most common used reference systems are principally those adopted in terrestrial measurements. The geocentric datum present a geometric nature and is now days used in its three dimensions with the possibility of determining the elevation respect to the ellipsoid. This measurement are affected by a very low value of uncertainty due to the extremely accurate phase positioning.

The strong point of the ellipsoid is its materialization directly during the survey, on the other hand its weak point is to be a general geometric reference system with pour connections with the field of gravity, that instead influences the level of the surface water.

The vertical datum is the materialization of the geoid from the physic point of view. It is defined as an equipotential surface materialized through gradients and gravity combined measurements.

This surface is commonly coinciding with a single point which will have a certain potential W0 (zero level), and all elevations are referred to this point.

This definition gives to the elevation value a physical meaning, but not directly connected to the coastal sites. In- deed the elevation measured on the geoid is only remotely connected to the local water position, because the zero height point is conventionally taken as the average of the sea level (MSL) and also because the zero height point is generally far from the local site of interest.

The Geoid is not sufficient by itself to describe the Coastal Zone, because reference that takes into account where is the water in the area of interest is needed too.

The averaged surface of the conventional sea does not describe the local trend of the sea level, from the spatial and chronological point of view. For this reason the reference mast be founded in the MSL, measured locally in the area of studies and calculated over an enough long period of time.

That level, we will call the Local Mean Sea Level (LMSL, presents variables differences with the ellipsoid be- cause of the geometric nature of it, but also with the geoid, for the reasons mentioned above. Those differences and its modelling and the measurement uncertainty must be estimated continuously on the whole coast. This aspect should be taken into account to define quality of the measures.

Tidal Datum can be connected at the LMSL: it is a vertical reference related to the astronomical tide. A tidal measurement frame should be installed along the national coast to monitoring the sea water level trough a net of onsite sensors. To define the vertical Datum along the coast a connection between the Tidal measurement frame and geoid (physic) and ellipsoid (geometric) is mandatory.

\subsection{The study of the transition between Land-Air- Water}

The Coastal Zone ${ }^{2}$ is mainly composed by three different matter states, one gassy (atmosphere), one liquid (water) and one solid (land/ ice).

Most of human activities are concentrated on the emerged area and the description of the edge between water and land and its position in the space assumes relevant importance: the set of point of the water edge where water meet land. Being the result of the intersection between two surfaces, the coastline practically could be considered as continuous line. This line isn't planar, because both the land and water surfaces generally don't have a planar or regular profile. Their continuous movements over time produce variation of the intersection line between land and sea (e.g. tectonic, volcanic phenomena, such as subsidence and bradyseism and sedimentary process erosive and deposition- al).

Between the infinite, irregular and variable interfacing lines, the European INSPIRE Directive defines it as:

a) Shoreline :"is a boundary where a Sea Area meets land. However there can be many different shoreline Depending on the tidal state. A Shoreline Therefore must have a value for the water level that Identifies the tidal been used to define the shoreline ".

b) Coastline : "is specialization of shoreline When the water level is equal to the MHW. A Coastline will be Typically the spatial object type used for most applications that need to show a sea / land boundary".

The above definitions describe the vertical position of the sea surface related to the tide phenomena, considering the variation of the profile due to the meteorological conditions in the examined area.

Also the variation of the earth surface position are considered, due to a sedimentary dynamic which process are characterized by sediments spatial and temporal

\footnotetext{
${ }^{2}$ It is the sector where five spheres are combined (atmosphere, hydrosphere, cryosphere, biosphere e geosphere) highlighting the complexity of the process. The edge between water and land is important to define what is emerged (land) and what submersed (Sea Bottom).
} 
variations through the movements between emerged and submersed part of the coastal zone (transversally and longitudinally to the littoral zone : e.g. sandy beach which change season- ally the profile in relation with the coastal hydro-dynamicity and sedimentary balance): for this reason the survey methods will depend on the time scale selected.

The identification on the shore line is made in two distinct phases:

- Shoreline character indicator selection and definition (eg. Wet-dry sector, surface at HWL (High Water Level) or LWL (Low Water Level), dunes systems).

- Choice of Shoreline character indicator based on the available data (homogeneity, resolution and spatial- temporal value).

Many authors highlight that the most significant, and potentially not correct assumption of shoreline data, is to attribute to the instantaneous shoreline the validity of medium conditions shoreline.

In the horizontal plane analysis, e.g. by projecting the surveyed shore or coast line, point by point to represent it on a chart, the represented planar line (shoreline) will be affected by the variability and validity of the considered zone (it will represent a continuous line with a buffer of variability, which also depend by the representation scale - Figure 1).
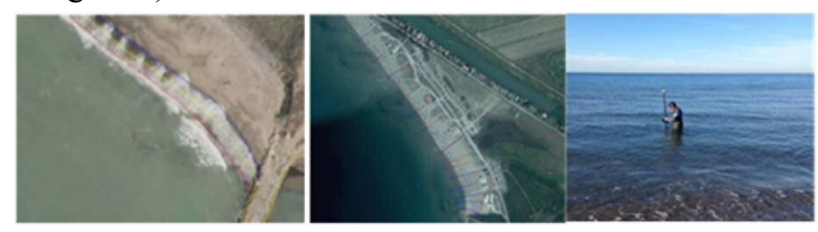

Figure 1 - Example of Shoreline survey with mixed methodology

\section{Measurement of the instantaneous shoreline}

Based on the consideration made in previous paragraph, the interfacing line between water and land assumes different definitions and above all change over time. The only line that is directly surveyed is the one obtained by measurement of shore line in a certain moment: the instantaneous shoreline.

This line should be generalized and referred to the INSPIRE definition of shoreline and coastline. The methodology of survey to define the instantaneous shoreline are mainly three:

a) Direct : through the direct measurement survey in the topographic field with classical equipment and standard procedure. Theoretically this survey is more accurate but more time and more resources are needed: this methodology is often applied for case study or test areas.

b) Indirect : this methodology is typically connected to remote sensing, where data are collected by remote platform not directly located on the area of survey.

c) Mixed : land and water are studied separately and then are combined; this methodology is based on describing land and seabed morphology as a continuum and on the extraction of the shoreline or coastline, measuring the water level with an independent methods (e.g. tidal gauge net).

Direct surveys methodology gathers tridimensional spatial coordinate and also collects the time of the surveyed points. Remote sensing (indirect) have often a bi-dimensional approach to the measure, which can be easily converted in tri-dimension introducing the spatial variation of the coastline and its uncertainty assessment.

For example if the tidal effects in a specific area produce a 1 meter maximum planimetric variation of the shoreline, $\mathrm{a}+-1$ meter buffer overlapping the coastline can be represented.

\subsection{Geometric tracking of the shoreline}

The ideal conditions for the tracking of the shoreline using aerial photography is calm sea, without waves. In this case only the sea-water transparency would represent an uncertainty in identifying clearly the water-land boundaries. With the presence of waves, some consideration have to be done. Approximately cylindrical and sinusoidal waves are symmetric to the water level and theoretically this would be verifiable in deep water and in dead sea conditions. Approaching the coast, waves are influenced by the sea bottom with refraction, changing in shape (shoaling) but their liquid particles still maintain their orbital motion, without mass flux (Figure 2).

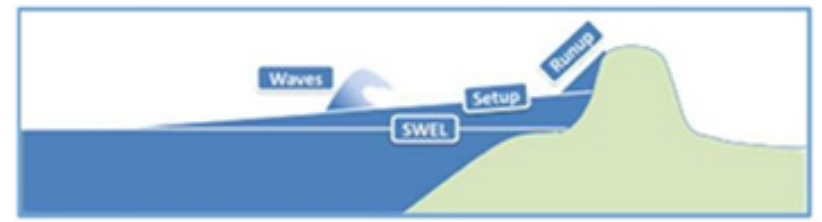

Figure 2 - The waves dynamics near the shoreline

In this zone called Surf-zone waves nor can be assumed as sinusoidal and symmetric to then mean of instantaneous calm sea level (Figure 3- still water elevation $\mathrm{SWELL}^{3}$ ).

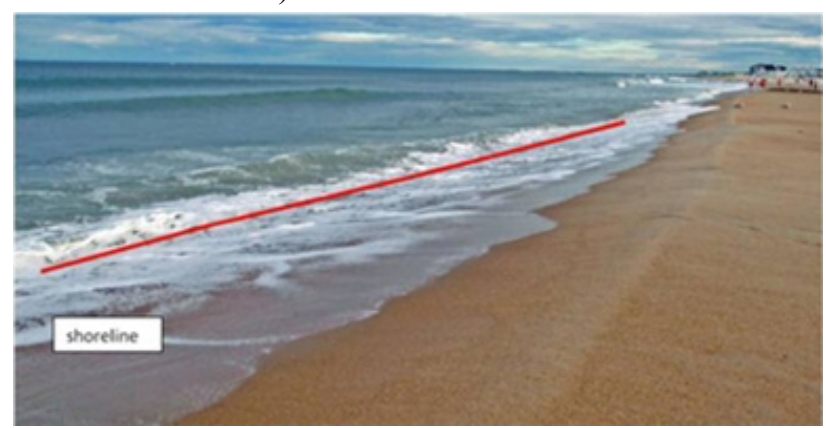

Figure 3- Example of the SWELL line- red line in Picture

The flux, and the equivalent reflux, of water generated by the waves breaking produces a raising on the sandy beach (wave setup) which is to be taken into account during the shoreline tracking.

\footnotetext{
${ }^{3} \mathrm{http}: / / w w w . f e m a . g o v / c o a s t a l-f l o o d-r i s k-m a p p i n g-$ process
} 
Looking at orthophotos of a shore where the waves break, the last breaking wave for the wave setup, is generally located close to the line obtained by the intersection between the SWELL and the beach (red line in the Figure 4).

In conclusion, excepted for the highest waves, a breaking wave reflux generally is not able to draw back (seaward) significantly the instantaneous shoreline from the SWELL.

In such circumstances the last breaking wave before runup represent a good cartographic interpretation reference for shoreline tracking in rough sea conditions.

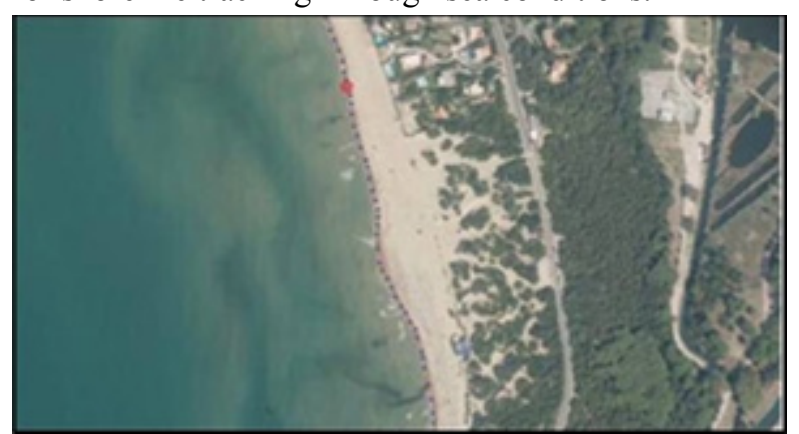

Figure 4 - Example of Shoreline digitized from AGEA 2014 orthographic Image

Based on the references above explained the tracking and extrapolation of the Lazio region shoreline, including the Pontine islands, were obtained by extraction of the digitized line (coloured in the picture below) orthophotos AGEA 2014 (resolution 0.20 meters, flight 2014).

The shoreline is highly variable by its nature and this fact dependent on many factors such:

- vertical variations in the average sea level (tides)

- wave motion

- morphology and lithology of the slope of the beach and shoreline.

It is evident that drawing a single line from aerial photos has intrinsically qualitative limits, due to a bidimensional approach.

With reference to the paragraph 2.1 definitions, it is clear that when only an orthophotos is available, it is possible to define a shoreline corresponding to a mean sea level closely to the instant of the photo and generalize it to a scale that takes into account the dynamicity of the coast.

\section{Shoreline Codification (DPCM 10 Nov. 2011 - IHO S57)}

With the coming into effect of the Prime Ministry Decree (DPCM) dated on 10th November 2011 "Technical rules for the definition of the geo-topographic databases content specifications", all the geo-topographic databases representing the base for territorial information for public administrations and for the spatial data collection and management, in Italy have to be compliant with a specific frame.

The DPCM 10/11/2011 refer to the scope and general principles at the national level defined by the Directive INSPIRE (Infrastructure for Spatial Information in the European Community).
Annex 1 of the Prime Ministerial Decree "catalogue of the spatial data - content specifications for Geotopographic Database " identifies which are the spatial data used to represent and describe the territory to natural and anthropic aspects. The information are organized in layers, themes and classes, with relationships and constraints between the data itself.

The reference frame "Class" defines the representation of a specific type of spatial objects, while the layers and the themes are used only to collect classes in morphologically and functionally homogeneous subsets.

Article. 59 of Legislative Decree. N. 82/2005 "Digital Administration Code" has established the National Repertory of Territorial Data (RNDT) whose aim is to "facilitate the sharing of general interest data by public authorities at national, regional and local level. "

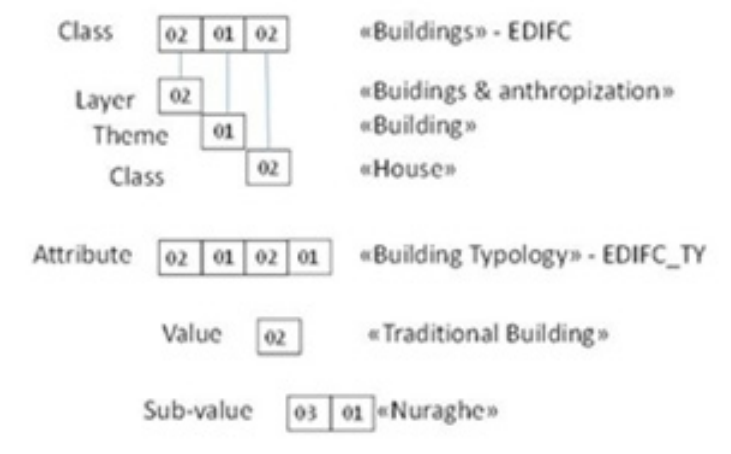

Table 1 - RNDT codification Table

The RNDT is a metadata catalogue based on ISO Standard 19115: 2003, 19119: 2005 and 19139: 2007 and com- pliant with the European Directive 2007/2/EC (INSPIRE) and with Regulation (EC) No. 1205/2008.

Classes and attributes are encoded following two different mode, an alphanumeric one, to simplify the recognition of the class or the attribute to which they refer, and a numeric one.

Port constructions are described within the "LAYER 02 Buildings and construction - THEME - Defence and hydraulic drainage constructions 0205":

1)In Class "Navigational facilities (AT_NAV 020,504)", there are structures such as, dry docks, floating docks, ramps etc.

2)The Class "Port constructions and coastal defence (OP_POR - 020505)," includes the shoreline construc- tion for defence in front of an harbour whose aim is to protect the coast from the water waves, the port in- frastructures such as piers, docks, and the constructions for coastal defence as breakwaters.

Outside the port, coastline is described in the "LAYER 04 - Hydrography - THEME Marine Waters 0402", in the Class "Cartographic Marine Coastline (CS_MAR 040201)."

In DPCM the THEME "Marine Waters 0402" includes all the classes of objects that define the cartographic 
shoreline (it is an ideal line where land and sea meet each other, generally derived by photogrammetric and refers to MSL. As defined in the directives, it is a conventional line, and it can include, for example, land areas (islands), nat- ural land such rocks / rocks or sandy areas / dunes, and coastal defence such breakwaters, dams, etc ,.

The theme "Hydraulic constructions, defence and hydraulic drainage " is defined as follows:

"Barrage Construction in front an harbour with the function to protect the coast from the water waves. This Theme includes all the entities that are in charge of control, retention and access in the land-water interactions. It includes the harbour constructions such as docks, piers, and the coastal defence as breakwaters etc ...All the elements are merged into one class because they identify entities with multiple functions (e.g. breakwater ... ) ".

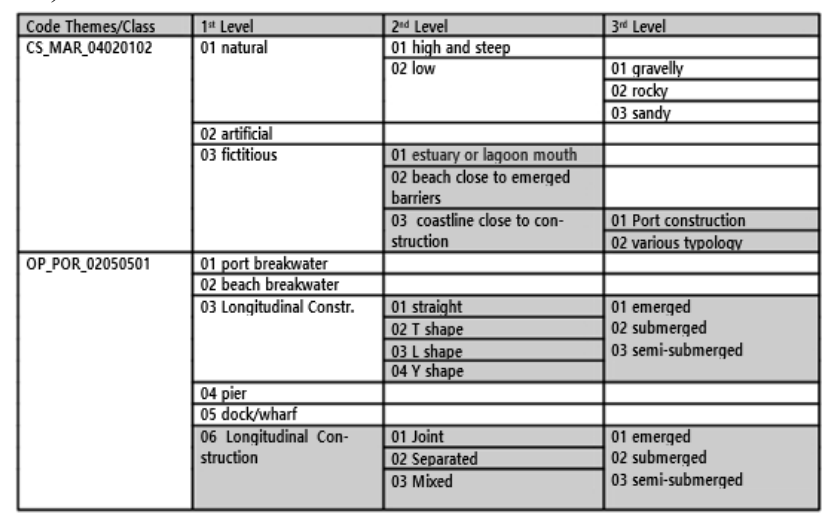

Table 2 - Scheme compliant with DPCM 10/11/2011

According with the definition of the DPCM 10/11/2011 it was decided to use a simplified relational scheme with the introduction and implementation of the following fields: "IHO S-57 Transfer Standard for Digital Hydrographic Data" that is the standard reference for exchange of hydrographic data, including their publication as ENC (Electronic Navigational Chart).

In the S-57 data model various entities are defined through "Feature Object" that contain descriptive attributes, and "Spatial Object" which define the geometry of the feature.

An S57 schema is represented in figure 5.

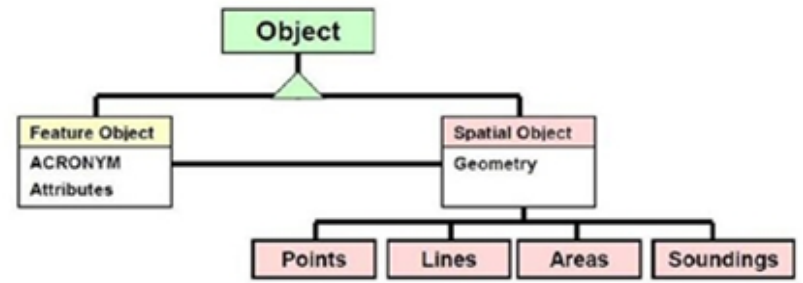

Figure 5 - IHO S-57 Structure scheme

Based on the S-57 standards, it was decided to re-encode the shoreline maintaining much of the geometry already used for encoding it in compliance with DPCM geotopographic database standard.

Following, some example of natural and artificial shorelines and their related encodings.

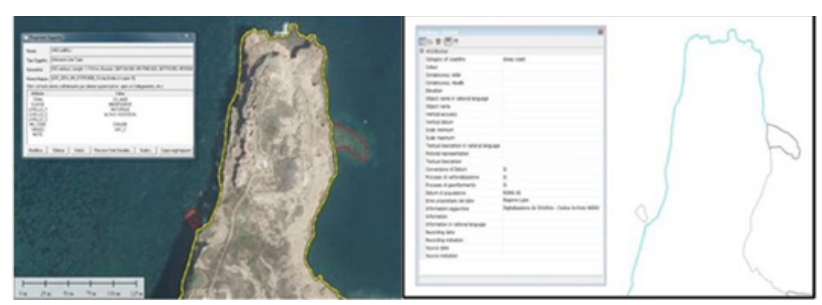

Figure 6 - Ventotene Island - Example of coding - CS_MAR 040201 - DPCM 10/11/2011- Coastline - IHO S-57

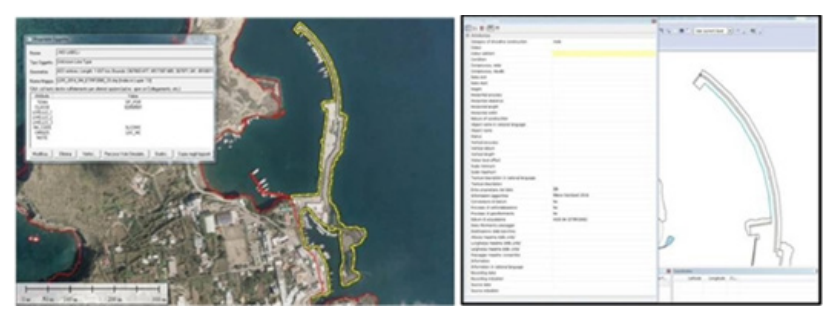

Figure 7 - Ventotene Island - Example of coding - OP POR 020505 - DPCM 10/11/2011- Shoreline construction - THO S57

\section{Conclusion}

The collaboration between the Navy Hydrographic Institute and the Lazio Region, within the project "POR ERDF 2007-2013 INTERCOAST" has led to the definition of the shoreline of the Lazio region through the analysis and digitalization of AGEA 2014 orthophotos.

The shoreline defined jointly by the IIM and the Lazio Region Authority and exchanged for their respective uses, was coded in two different standard methodologies that have much in common. The results has been stored in the own databases and the standard encoding will allow the use of data for different studies or applications, assuring the complete data sharing and interoperability.

The work so far described, especially for the operational aspects related to the tracking and encoding, compliant with to the National Directive on Geo-topographic Database, has been taken into account as guideline to the work table on coastal erosion of the Environmental Ministry.

The study of the morphology of the coastal zone through the digitizing of an orthophoto is one of the widest methodology used for describing the shape of a natural coastline. In fact this approach appears by far cheaper than surveying beaches and islands with a classical geotopographic team (boots on the field). The natural coastline also has a large geographical extension and no particularly high accuracy is needed.

However in that applications where more accuracy is needed, such as artificial coastline (harbour and port), maritime engineering, the direct methodology is preferred References should be cited in the text, thus (Smith, 1987b), and listed in alphabetical order in the reference section.

\section{References}

European Commission (2013), INSPIRE Infrastructure for Spatial Information in Europe - Data Specification on Sea Regions - Technical Guidelines 
IHO (2000), S 57 - IHO Transfer Standard for Digital Hydrographic Data

CNIPA, the National Repertory of Territorial Data (RNDT) "Specifics for Geo-topographic DataBase"

Boak E.H., Turner I.L., 2005. Shoreline Definition and Detection: A Review. JCR, 16(4), 688-703.

Davis R.J., Fitzgerald D., 2004. Beaches and coast. Blackwell ScienceLtd. EEA 2006 The changing faces of Europe's coastal areas. European Environment Agency, Copenhagen.

Lavalle C., Rocha Gomes C., Baranzelli C., e Silva F.B., 2011. Coastal zone.Policy alternatives impacts on European coastal zone 2000- 2005. Joint Research Centre Technical Note, EU.

Portman M.E., 2016. Environmental planning for Oceans and coasts: methods, tools and technologies. Springer. UNEP, 2006. Marine and coastal ecosystems and human well-being: a synthesis report based on the findings of the Millennium Ecosystem Assessment.

I.I. 3176 Disciplinare Tecnico per la Standardizzazione dei Rilievi Idrografici Ed. 2016

\section{I.I. 1028 Norme di Massima per l'Attivita' IDROGRAFICA (NORMAS) Ed. 2016}

Decreto della Presidenza del Consiglio dei Ministri 10 novembre 2011 ( Geodetical National Refererence System : ETRF 2000 - at 2008.0)

IHO S-32 - Hydrographic Dictionary

IHO S-44 - IHO Standards for Hydrographic Surveys (5th edition) 2008

IHO S-57 - IHO Transfer Standard for Digital Hydrographic Data, Edition 3.1, November 2000 - Main Document IHO M2 - The need for National Hydrographic Services

IHO C13 - Manual on Hydrography, 1st Edition, (Correction Feb. 2011) 